\title{
A Framework of Personality Cues for Conversational Agents
}

\author{
Rangina Ahmad \\ TU Braunschweig \\ rangina.ahmad@tu-bs.de \\ Ulrich Gnewuch \\ Karlsruhe Institute of Technology \\ ulrich.gnewuch@kit.edu
}

\author{
Dominik Siemon \\ LUT University \\ dominik.siemon@lut.fi \\ Susanne Robra-Bissantz \\ TU Braunschweig \\ s.robra-bissantz@tu-bs.de
}

\begin{abstract}
Conversational agents (CAs)-software systems emulating conversations with humans through natural language-reshape our communication environment. As CAs have been widely used for applications requiring human-like interactions, a key goal in information systems (IS) research and practice is to be able to create CAs that exhibit a particular personality. However, existing research on CA personality is scattered across different fields and researchers and practitioners face difficulty in understanding the current state of the art on the design of CA personality. To address this gap, we systematically analyze existing studies and develop a framework on how to imbue CAs with personality cues and how to organize the underlying range of expressive variation regarding the Big Five personality traits. Our framework contributes to IS research by providing an overview of CA personality cues in verbal and non-verbal language and supports practitioners in designing CAs with a particular personality.
\end{abstract}

\section{Introduction}

Conversational agents (CAs) are reshaping our communication environment by emulating conversation with humans through natural language [1]. From the initial stage of rule-based chatbots to the era of rapid development in artificial intelligence (AI), CAs have become increasingly capable of handling highly complex tasks with human qualities $[2,3]$. Various types of CAs have emerged and are used in a variety of different application domains (e.g. customer service, mental health care, education) $[4,5]$. With their growing popularity, much research in information systems (IS) has been dedicated to the design of CAs $[6,7,8,9]$. Creating a $\mathrm{CA}$ is, by its nature, multidisciplinary and requires the application of a variety of disciplines ranging from agent systems and interface design to sociology and psychology [10].
Hence, both technical as well as social aspects are considered vital when designing CAs in order to manage successful interactions with human beings $[11,12]$.

In recent years, researchers have specifically focused on designing human-like CAs by giving them a personality: first, to propose more complete cognitive models of CAs, and second, to propose CAs capable of sustaining more human-like interactions with people [13, 14, 15]. The Big Five model of personality has emerged as a standard in psychology, with research over the last fifty years systematically documenting correlations between personality traits and a wide range of behaviors $[16,17,18]$. The concept of personality is primarily of interest for CA designers because of the ways in which it affects actual behavior, and precisely since those behaviors are communicative, they establish a channel of social interaction crucial to the smoothness and effectiveness of a conversation [19,20]. The domain of verbal and non-verbal language in which information indicative of personality traits can be expressed is large and diverse and contains modalities such as joking, speaking in a deep/high-pitched voice, holding a gaze and gesturing [21, 22], all of which can be further divided into numerous features [23]. Research has shown, that people not only prefer CAs that align with human behavior, such as by speech style or mimicking head movements [24], but are also increasingly attracted to CAs that adapt their personality to the human over time rather than maintaining a static or consistently similar personality [24].

While CA personality has been researched extensively in human-computer interaction (HCI) and a variety of studies have addressed multiple aspects of CA personality, existing research is scattered across different fields. Consequently, researchers and practitioners face difficulty in understanding the current state of the art on the design of CA personality. To address this gap, we develop a framework on how to imbue CAs with personality cues and how to organize the underlying range of expressive variation 
regarding the Big Five personality traits. We aim to provide an overview of personality markers manifested in verbal and non-verbal language and derive implications for future studies with the following research question:

\section{What are existing personality cues for conversational agents and how to structure them?}

To answer this research question, we conduct a systematic literature review (SLR) following the guidelines by Webster and Watson [25]. In order to identify existing personality cues in CAs, we analyze 22 publications and structure our review using a framework based on the Big Five model of personality. Our study contributes to IS research by providing a comprehensive overview of personality cues specific to the Big Five dimensions, divided into verbal, paraverbal and body language features. The systematic framework of personality cues can be used by practitioners to implement and test their effects in the design of their CAs.

The paper is structured as follows: First, we introduce the foundations of personality theory and CAs before presenting our SLR, the results of our framework and the discussion of them, and ultimately, the paper's contribution and limitations. The paper concludes with implications for further research.

\section{Research Background}

\subsection{The Big Five Model of Personality}

In psychology (specifically in trait theory) it is assumed that people's behavior and feelings can be explained to some extent in terms of underlying personality traits, which are regarded as specific features of one's personality and are enduring dispositions that are relatively stable over time [17, 18]. Multifactorial models such as the generally accepted taxonomy of the Big Five model have formalized and standardized personality traits in order to measure personality [26]. Compared to other existing personality models, the Big Five model has been found to be stable across cultures, as well as observers and has been widely used in a variety of disciplines [18]. Derived through factorial studies, five fundamental traits or dimensions have been described and defined for a comprehensive assessment of individuals: extraversion, openness (to experience), agreeableness, conscientiousness and neuroticism [17, $18]$.

The trait openness (to experience) refers to the extent to which a person is open to experiencing a variety of activities, and prefers novelty over convention [27]. It manifests in traits such as creativity, imaginativeness, curiosity, and aesthetic appreciation. Open people are heavily invested in cultivating new experiences, and are described as original, curious and liberal [28]. People who fall at the other end of this factor - called the opposite pole are uncreative, conventional and narrow in their interests, conservative and sometimes rigid in their approach to life's challenges and opportunities [27]. The second dimension - conscientiousness - refers to the extent to which people prefer an organized or a flexible approach in life, and is thus concerned with the way in which they control, regulate, and direct their impulses [28]. People who score high on conscientiousness are hardworking, conscientious, punctual, and persevering [27]. People who score low on conscientiousness, in contrast, tend to be disorganized, negligent, lazy, aimless and are likely to give up [29]. The third Big Five trait extraversion is the orientation to the outside world rather than to private experience. The factor thus refers to the extent to which people enjoy company, and seek excitement and stimulation. People who score high on extraversion tend to be affectionate, jovial, talkative, joiners, and fun-loving [27]. In contrast, low extraversion (also called introversion) scorers are likely to be reserved, quiet, loners, passive, and lacking the ability to express strong emotion [29]. The dimension agreeableness reflects individual differences concerning cooperation and social harmony [29]. Individuals who score in the direction of agreeableness tend to be trusting, generous, softhearted, acceptant, and good-natured. People who score in the other direction are generally suspicious, stingy, unfriendly, irritable, and critical of other people [28]. Lastly, the fifth dimension, neuroticism, has to do with people's emotional instability. Although neurosis is an almost obsolete psychiatric term referring to pathological manifestations of anxiety, neuroticism refers to a considerably wider range of negative emotions, including anger, sadness, shame, and embarrassment [27]. People scoring high on neuroticism can be characterized as being anxious, nervous, moody, and vulnerable [27]. On the other hand, people scoring low on neuroticism can be described as emotionally stable, calm and eventempered [28]. The Big Five personality traits are summarized in table 1. 
Table 1. The Big Five Personality Traits

\begin{tabular}{|l|l|l|}
\hline Dimension & High Scores & Low Scores \\
\hline Openness & $\begin{array}{l}\text { imaginative, creative, } \\
\text { original, prefers } \\
\text { variety, curious, } \\
\text { liberal }\end{array}$ & $\begin{array}{l}\text { down-to-earth, } \\
\text { uncreative, } \\
\text { conventional, } \\
\text { conservative }\end{array}$ \\
\hline Conscientiousness & $\begin{array}{l}\text { hard-working, well- } \\
\text { organized, punctual, } \\
\text { ambitious, } \\
\text { persevering }\end{array}$ & $\begin{array}{l}\text { negligent, lazy, } \\
\text { disorganized, } \\
\text { late, aimless, } \\
\text { quitting }\end{array}$ \\
\hline Extraversion & $\begin{array}{l}\text { affectionate, joiner, } \\
\text { talkative, fun loving, } \\
\text { active, passionate }\end{array}$ & $\begin{array}{l}\text { reserved, loner, } \\
\text { quiet, sober, } \\
\text { passive, unfeeling }\end{array}$ \\
\hline Agreeableness & $\begin{array}{l}\text { soft-hearted, trusting, } \\
\text { generous, } \\
\text { acquiescent, lenient, } \\
\text { good-natured }\end{array}$ & $\begin{array}{l}\text { ruthless, } \\
\text { suspicious, } \\
\text { stingy, } \\
\text { antagonistic, } \\
\text { critical, irritable }\end{array}$ \\
\hline Neuroticism & $\begin{array}{l}\text { anxious, tempe- } \\
\text { ramental, self-con- } \\
\text { scious, emotional }\end{array}$ & $\begin{array}{l}\text { calm, even- } \\
\text { tempered, } \\
\text { comfortable, } \\
\text { unemotional }\end{array}$ \\
\hline
\end{tabular}

\subsection{Personality and Linguistic Cues}

The Big Five approach to the taxonomy of traits is based on natural language, more precisely lexical resources [13]. The lexical hypothesis states that most of socially relevant and salient personality characteristics have become encoded in the natural language [30]. Correlations between a range of linguistic variables and personality traits have been discovered by the frequency with which certain categories of words are used as well as the variations in word usage [31, 32]. Language use has been therefore scientifically proven to be unique, relatively reliable over time and internally consistent [30]. Accordingly, language constitutes and predicts aspects of personality. Psychologists have further discovered that people use a variety of cues depending upon the context [16]. However, to guide the determination of personality, people consistently rely a great deal on verbal and non-verbal cues. Mehrabian et al. [33, 34] developed a rule in the communication process, stating that personality can be expressed through verbal language (words), paraverbal language (tone of voice, pitch, volume, pace, etc.) and body language (gestures, posture, facial expressions, etc.). For example, in paraverbal language, cues such as speech rate range, loudness or fundamental frequency distinguish submissive from dominant individuals [35]. On all three communication levels, such language cues can be used to help imbue CAs with personalities, in order to sustain more human-like interactions with people [13, 14, 15]. A combination of these linguistic cues can further help portray a specific CA personality: as some contexts, for example in mental health care, require CAs to have a more emphatic and agreeable personality rather than a neurotic personality. Understanding how to not imbue a CA with specific cues in some use cases can therefore be helpful, too.

\subsection{Conversational Agents}

CAs are software systems designed to interact with humans using natural language [8]. The first CA, called ELIZA, was developed in 1966 [36] and although it was a rule-based system, it was already able to mimic human conversations and create perceptions of personality among their users. Since then, technology has immensely improved due to advances in $\mathrm{AI}$ and machine learning and CAs are implemented in many different application domains such as customer service, mental health care, and education $[4,5]$. In doing so, CAs use modern natural language processing (NLP) techniques to be able to understand their users, but also to communicate in a natural way. Diederich et al. [9] state that CAs can take various forms and differ by communication mode, embodiment and the context in which they are used. Since CAs are used to interact or communicate, the mode of communication and embodiment determine the type of social cues that can be used to facilitate rich communication $[7,37]$. These characteristics, i.e., the use of social cues via embodiment or communication mode, reflect how a CA is perceived by its users [38]. Basically, three types of CAs can be distinguished: they differ in their embodiment and communication mode, which are text-based CAs (i.e., chatbots), embodied CAs (e.g., avatars or 3D animated figures) and voice-based CAs (e.g., Amazon's Alexa). Chatbots, i.e. text-based CAs, can primarily communicate via their textual language, while voicebased CAs are considered a richer medium due to their additional use of voice [9]. They can transmit more information than a pure text-based chatbot. Another form of CAs are embodied CAs (ECAs), which additionally have a visual form, e.g. an animated 3D model and can therefore use body language to transmit further information to their users [38, 39]. In combination with a voice, an ECA represents a rich medium that can transmit a range of social cues [40, 41]. For example, hand gestures in combination with a defining and louder voice can transmit more information than a pure text-based chatbot [35]. These social cues consequently influence the way the user perceives the CA, for example what kind of personality the CA reflects due to the imbued cues [7, $15,37,42,43]$. 
However, it has been shown that the design of CAs is not only a technical challenge [7], but that psychological factors, which may arise from the choice of cues, must also be considered. This is because many users attribute a personality to a CA, which is triggered by the use of various verbal, paraverbal, or body language cues. In order for designers and providers of CAs to better understand the effect that certain cues have on the perception of personality of their CAs, it should be clearly shown which cues can be used for which type of CAs to induce which personality traits.

\section{Method}

To identify specific cues for the manifestations of personality in CAs, we conducted a systematic literature review following the approach of Webster and Watson [36]. We decided to use two databases from different disciplines (i.e., Scopus and ACM Digital Library) to cover a broad range of studies in IS and HCI. Next, we specified the search term, that we constructed to correspond to our focus on CAs and personality and included synonyms for each term. We identified the following terms that are used for any forms of CAs: conversational agent, virtual agent, digital agent, conversational assistant, virtual assistant, digital assistant, chatbot or chatterbot. We combined these terms with the search criteria dealing with personality traits and came up with the following search term in total: ABS(("conversational agent" OR "virtual agent" OR "digital agent" OR "conversational assistant" OR "virtual assistant" OR "digital assistant" OR chatbot* OR chatterbot* ${ }^{*}$ OR chatterbox*) AND (personality OR "big 5" OR "big five" OR "openness to experience" OR "conscientiousness" OR "extraversion" OR "agreeableness" OR "neuroticism")).

The search was conducted in June 2020 and the search string was used to search the articles' abstracts, resulting in 142 documents for the ACM digital library and 285 for Scopus (Scopus includes the AIS eLibrary). After reviewing abstracts, 335 articles were excluded due to limited relevance or lack of scientific rigor, leaving 92 articles that were analyzed based on their full text. After a full text analysis of the remaining papers, 66 papers were excluded leaving 18 papers for a qualitative content analysis. Based on these papers, a forward and backward search was performed, resulting in the inclusion of an additional 4 papers. This selection was based on a consensus agreement reached iteratively in a discussion between the authors. Thus, in total, 22 relevant papers were examined (see figure 2). The criterion for relevance was that the paper must cover at least one or more personality traits (Big Five) and apply a defined cue to any form of CAs (e.g. digital assistant, chatbot, etc.). In addition, the paper must describe the effect of a design feature/cue on the CA's perceived personality. For example, Neff et al. [21] was found via the Scopus database, covers an ECA, various cues for verbal language and body language regarding the personality trait extraversion.

We followed a deductive qualitative content analysis based on the aspects related to the selection criteria. First, we looked at what personality traits were considered and coded the traits according to the Big Five dimensions. Next, we examined the form of the CA (chatbot, voice assistant or ECA), and based on that, we identified the incorporated features or social cues and coded the characteristics verbal language, paraverbal language, and body language. The last step was to identify what effect the cue(s) had on the defined personality trait, e.g., reflecting a high degree or low degree of the trait. Figure 1 provides an overview of our systematic literature review.

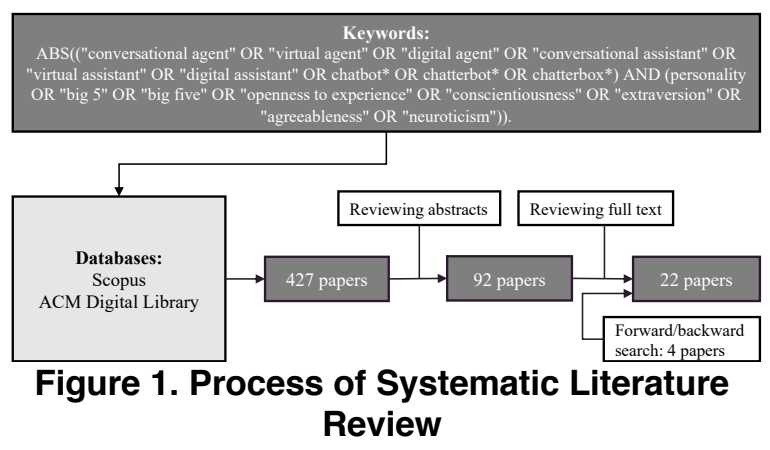

\section{Results}

Out of the 22 papers that we analyzed to derive existing personality cues from, 14 studies focused on ECAs, while 4 studies looked more closely at chatbots and another 4 at voice assistants. The papers were published between 2000 and 2020, the majority of them using experiments as their methodologies. Based on each of the Big Five personality dimensions [17, 18], we classified the cues into the three categories verbal, paraverbal and body language $[33,34]$. In total, we identified 148 cues. The results showed that the fewest cues were found in the dimension openness (for experience), while the trait extraversion had the most cues on both its poles. In the following, we present the findings of our personality cues in detail.

Openness (to experience). This personality trait was the dimension with the smallest number of cues (a total of 6) that we have found in our body of research. While cues such as wrist extension, more pronounced movements, increased stroke scale and more 
controlled body movements [22] reflect a high score in openness when it comes to an ECA's body language, the studies did not contain any further information on the trait's opposite pole. In the category paraverbal language, research showed that disfluencies in speech [46] is characteristic for low openness, while high emotionality in female voice [47] is considered a personality cue for high openness. We did not find any cues regarding verbal language.

Agreeableness. While we could not derive any paraverbal language cues for this dimension, the findings of our SLR did result into a number of body and verbal language cues. As for the latter, a CA can be imbued with verbal cues such as high verbosity, verb strength, content polarity [48] and affective expressions [49] in order to let the CA appear more agreeable. Further, by making more restatements [48] and suggestions [49] and asking more questions [49], a CA can also convey agreeableness. However, assertions, projective statements and terse expressions [49] let a CA appear less agreeable. When it comes to body language, cues such as a tilted head [48] and exhibiting less of a vertical arm [22] reflect high agreeableness, whereas average velocity, less arm swivels, body disfluency, less clavicle use, velocity warp and less controlled body movements [22] are found to be cues representing a low agreeable body language.

Conscientiousness. The only verbal cue found from the analyzed papers reflecting high conscientiousness is the frequent use of discourse markers (e.g. I mean, you know, like) [50]. No further information was found on how low conscientiousness could be reached by means of verbal language. However, the body language of a low conscientious ECA can be depicted by a less relaxed posture, body disfluency and a less lifted clavicle [22]. Also, disfluency in speech and fillers [46] portray a less conscientious CA. Slow speed and high emotionality [47] in voice are found to have a correlation with high conscientiousness - however, this only applies to the female voice [47].

Neuroticism. The majority of cues found for this trait in the studies focused on high neuroticism (rather than low). For example, reduction in fluency and making longer pauses before responding [16] are verbal markers for low emotional stability. For paraverbal language, a higher proportion of silence to speech and the presence of speech discontinuities [16] reflects high neuroticism. Furthermore, a male voice that speaks fast and has a low wordiness [47] has as well been linked to low emotional stability in behavior. As for the category body language, a number of cues have been associated with high neuroticism: fewer other-directed gestures and more self-directed gestures, more frequent shifts in posture such as leaning more forward or a more tense and stiff posture [23], as well as more non-signaling hand motions (e.g. scratch on the body), increased gaze aversion, decreased head height [16], more arm swivels, less controlled body movements, clavicle use and a higher velocity warp [22]. The only mentioned cue in the studies that is indicative of low neuroticism is a relaxed posture [22].

Extraversion. This trait not only has more linguistic cues than any other dimension, but also its opposite pole - introversion - has been found to have a lot of personality markers (combined a total of over 90 cues). For example, introverted paraverbal language is achieved by disfluencies and being more hesitant in speech [46, 51] and a suggestive tone [52]. An extraverted paraverbal language on the contrast is reached by speaking fluidly [51], louder, with a higher fundamental frequency, a higher frequency range, with a faster speech rate [35], a more soft and friendly voice [53] and a rather commanding tone [52]. As for the category verbal language, many of the cues express each end of the personality dimension, i.e. either extraversion or introversion in terms of low and high settings. For example, an extraverted utterance can be conveyed through high verbosity, whereas low verbosity is perceived as more introverted [19]. Further markers reflecting an extraverted language are for example many restatements, many request confirmations, many emphasizer hedges [19] and the use of strong, confident words and phrasing [52], less filled pauses, less negation [19], low syntax complexity or high content polarity [21] and small talk [54]. A CA with an introverted language style, by contrast, could be imbued with the following linguistic markers: high syntactic complexity, low number of acknowledgements [21], formal speaking style [55], many filled pauses [56, 57] many negations, less restatements, less request confirmations [22, 58] as well as with timid, unassuming statements [57]. Figure 2 in the appendix provides the framework for the completed list of both, extraverted and introverted verbal language cues derived from the SLR. As the majority of our analyzed studies focused on ECAs, the findings, thus, on extraverted and introverted body language cues is accordingly high (over 40 cues). For instance, introversion is reflected by low spatial extent [19], bringing the hands together in front of the body [59], low gesture rate, backward leaning and turning away [60], less use of the clavicle or a far body position to the interlocutor [22]. Extraversion in the category body language, however, is manifested for example in a forward chest and hands away from the body [60], a higher gaze amount [58], smiling [61], an intimate proximity level [56] and also when the elbows 
move away from the body during gesturing [23]. Furthermore, an extraverted ECA could be displayed by a more tilted head, a higher gaze amount and gestures with greater frequency [58]. The complete framework of body language cues for the dimension extraversion (introversion) and further cues for all five dimensions can be found in figure 2 in the appendix.

\section{Discussion}

The design of CA personality has gained widespread attention in research and practice [7, 9]. However, a comprehensive overview of what constitutes CA personality and how to design a CA to exhibit certain personality traits is lacking. Our research addresses this gap by systematically analyzing existing studies and developing a framework of personality cues for CAs. In total, we identified 148 cues that were classified into three categories and were based on the Big Five personality traits. Most cues can be found for the dimension extraversion (and its opposite pole introversion) these findings about personality cues of extraversion are in line with a large body of research, as this trait has received the most attention in linguistic studies $[31,62,63]$. Compared to extraversion, the remaining dimensions (specifically the traits openness to experience and conscientiousness) contain fewer cues, causing an imbalance in the framework. In the following, we discuss the contributions and implications of our work.

\subsection{Theoretical Contributions and Practical Implications}

The theoretical contributions of our work are threefold. First, our framework of personality cues for CAs provides a comprehensive overview of the current state of research on CA personality. While previous research has demonstrated that certain cues in the CA design lead to personality attributions among users [43], our framework offers a more holistic understanding of which cues have been used for which personality traits. This helps researchers to better position their work and identify future research opportunities. With our focus on personality cues, our framework differs from and extends previous research, such as the taxonomy developed by Feine et al. [7] or the classification provided by Seeger et al. [64], which examines and classifies social cues on a more general level without establishing a link to the concept of personality. Second, our framework sheds light on the differences between different personality traits and different types of cues. While extant research has focused on the personality trait of extraversion [63], mostly using verbal and body language cues, there are several blind spots in this research area. For example, our findings for extraverted paraverbal language cues were far less than those for the other two communication categories, and the dimension agreeableness having no paraverbal cues at all. In fact, cues for the category paraverbal language have been rather neglected throughout all five dimensions. Furthermore, the cues found for the personality dimensions openness (to experience) and conscientiousness are so few, that it might not be enough to create a holistic CA personality with them. While research on human-human interaction has shown that these types of cues also occur in verbal, paraverbal or body language, CA research has not investigated them so far. This implies that more studies are needed on personality traits such as openness (to experience) and conscientiousness, but also on agreeableness and neuroticism in order to close this research gap. Third, in our analysis, we also found that some results were not completely free of contradictions: some few cues have been found to both positively and negatively influence the manifestation of a certain personality trait. For example, using more filler words convey high conscientiousness in verbal language [50], however, less fillers in speech have as well been shown to correlate with low conscientiousness in paraverbal language [46]. This finding suggests that there are other contextual factors at play that shape users' personality perceptions of CAs. This highlights the need to take users' individual differences into account when studying CA personality.

Our research also offers interesting practical implications. First, CA designers can use our framework to analyze their own CAs and identify personality cues. This could help them to identify possible unwanted effects on the perception of their CA. Moreover, designers who aim to develop a new CA could use our framework to give the CA a very specific personality that corresponds to the organizations' image. For example, in a banking context, a different personality could be appropriate than in a shopping context. Our framework supports designers in translating these needs into concrete design decisions. For instance, a more formal language style could be used for a CA at a financial institution. Second, our framework highlights the need of practitioners to not only address the topic of $\mathrm{CA}$ personality on a superficial level. This is because even rather subtle cues, such as word fillers, longer pauses before responding, or a higher gaze amount can, for example, lead to perceptions of the $\mathrm{CA}$ as being more human-like. 


\subsection{Limitations}

As with any literature review, there are certain limitations to our work. First, although we followed established guidelines, our search strategy might have missed relevant publications. Since we focused specifically on CAs and studies that describe a clear relationship between a cue and perceived personality, a different approach might have resulted in a different framework. Moreover, due to the broad scope of our literature search, we identified a rather diverse set of papers from different domains. While some were more technical, other focused more on understanding user perceptions and treated the actual implementation of the CA as a black box. However, we believe that both approaches are valid and including their results increases the generalizability of our framework.

Second, to develop our framework, we drew upon existing classifications of cues $[33,34]$ and personality traits $[17,18]$. We acknowledge that there are different classifications for both, which would result in a different framework. However, we argue that the existing classifications that we used are suitable because they are well accepted theories and have been found to be stable across cultures as well as observers $[17,18]$.

\subsection{Future Research}

Our study also offers several promising directions for future research. First, our framework shows that there are several blind spots in existing research. More specifically, some personality traits and cues have not been studied yet, even though research from humanhuman interaction shows their importance. Therefore, future research is needed to fill these gaps and provide a more complete picture of what constitutes CA personality.

Second, one finding of our analysis was that there are a few inconsistent findings with regard to some cues. As noted before, this suggests that individual differences (e.g., users' own personality) may also play a role [15]. Future research could extend our framework to also consider these individual factors. In addition, more empirical research is needed to better understand the interplay between personality cues and individual user characteristics (e.g., personality, gender, cultural background).

Third, there are also numerous opportunities to apply our framework to the design of CA artifacts. For example, based on the identified cues, research could develop CAs that adapt their personality based on users' personalities and their individual preferences in communication style. Such adaptation could be performed automatically by the CA or manually by its user.

Finally, more interdisciplinary research is needed to better understand the ethical and societal implications of providing CAs with personality cues. While our framework illustrates that many cues have been found to lead to personality perceptions, such effects might not be appropriate or desired for all CAs. For example, these cues could also be used to unconsciously manipulate users in order to sell them products or increase their willingness to disclose personal information.

\section{Conclusion}

The aim of our research was to provide a framework of personality cues for CAs. Therefore, we conducted a SLR following the approach by Webster and Watson [25]. We analyzed 22 publications in the field of HCI and IS research and structured our framework based on the Big Five personality traits taxonomy. The identified cues manifesting each of the five personality dimensions were then classified into the three communication categories verbal, paraverbal and body language. Our framework contributes to IS research by providing an overview of personality cues manifested in verbal and non-verbal language and should be a useful resource for researchers and practitioners aiming to design CAs with a particular personality. While our analyzed set of papers contained a number of cues for all five personality dimensions, the results of our SLR also showed the imbalance of specific cues. Future research thus needs to fill these gaps and provide a more a complete picture of what exhibits CA personality.

\section{References}

[1] Luxton, D.D., "Ethical implications of conversational agents in global public health", Bulletin of the World Health Organization 98(4), 2020, pp. 285.

[2] Io, H.N., and C.B. Lee, "Chatbots and conversational agents: A bibliometric analysis", 2017 IEEE International Conference on Industrial Engineering and Engineering Management (IEEM), (2017), 215-219.

[3] Brendel, A.B., M. Mirbabaie, T.-B. Lembcke, and L. Hofeditz, "Ethical Management of Artificial Intelligence", Sustainability 13(4), 2021, pp. 1974.

[4] Gnewuch, U., S. Morana, and A. Maedche, "Towards Designing Cooperative and Social Conversational Agents for Customer Service", ICIS, (2017).

[5] Kocaballi, A.B., L. Laranjo, J. Quiroz, et al., Conversational Agents for Health and Wellbeing, 2020.

[6] Strohmann, T., D. Siemon and S. Robra-Bissantz, "Introducing the Virtual Companion Canvas - Towards Designing Collaborative Agents : Extended Abstract", 
Proceedings of the Workshop on Designing User Assistance in Intelligent Systems, Stockholm, Sweden, 2019. Ed.: S. Morana, 2019.

[7] Feine, J., U. Gnewuch, S. Morana, and A. Maedche, "A Taxonomy of Social Cues for Conversational Agents", International Journal of Human-Computer Studies 132, 2019, pp. 138-161.

[8] McTear, M., Z. Callejas, and D. Griol, The conversational interface: Talking to smart devices, Springer, 2016.

[9] Diederich, S., A. Brendel, S. Morana, and L. Kolbe, “On the Design of and Interaction with Conversational Agents: An Organizing and Assessing Review of Human-Computer Interaction Research", Journal of the Association for Information Systems, 2022.

[10] Isbister, K., and P. Doyle, "Design and evaluation of embodied conversational agents: A proposed taxonomy", The first international joint conference on autonomous agents \& multi-agent systems, Citeseer (2002).

[11] Araujo, T., "Living up to the chatbot hype: The influence of anthropomorphic design cues and communicative agency framing on conversational agent and company perceptions", Computers in Human Behavior 85, 2018, pp. 183-189.

[12] Go, E., and S.S. Sundar, "Humanizing chatbots: The effects of visual, identity and conversational cues on humanness perceptions", Computers in Human Behavior 97, 2019, pp. 304-316.

[13] Bouchet, F., and J.-P. Sansonnet, "Intelligent agents with personality: From adjectives to behavioral schemes", In Cognitively Informed Intelligent Interfaces: Systems Design and Development. IGI Global, 2012, 177-200.

[14] Grudin, J., and R. Jacques, "Chatbots, Humbots, and the Quest for Artificial General Intelligence", Proceedings of the 2019 CHI Conference on Human Factors in Computing Systems - CHI '19, ACM Press (2019), 111.

[15] Ahmad, R., D. Siemon, and S. Robra-Bissantz, "Communicating with Machines: Conversational Agents with Personality and the Role of Extraversion", Proceedings of the 54th Hawaii International Conference on System Sciences, (2021), 4043.

[16] Neff, M., N. Toothman, R. Bowmani, J.E.F. Tree, and M.A. Walker, "Don't scratch! Self-adaptors reflect emotional stability", International Workshop on Intelligent Virtual Agents, Springer (2011), 398-411.

[17] Goldberg, L.R., "An Alternative 'Description of Personality': The Big-Five Factor Structure",

[18] McCrae, R.R., and O.P. John, "An introduction to the five-factor model and its applications", Journal of personality 60(2), 1992, pp. 175-215.

[19] Hanna, N., and D. Richards, “The influence of users' personality on the perception of intelligent virtual agents' personality and the trust within a collaborative context", International Workshop on Multiagent Foundations of Social Computing, Springer (2015), 3147.
[20] Ball, G., and J. Breese, "Emotion and personality in a conversational agent", Embodied conversational agents 189, 2000.

[21] Neff, M., Y. Wang, R. Abbott, and M. Walker, "Evaluating the Effect of Gesture and Language on Personality Perception in Conversational Agents", Intelligent Virtual Agents, Springer (2010), 222-235.

[22] Smith, H.J., and M. Neff, "Understanding the impact of animated gesture performance on personality perceptions", ACM Transactions on Graphics (TOG) 36(4), 2017, pp. 1-12.

[23] Tolins, J., K. Liu, Y. Wang, J.E.F. Tree, M. Walker, and M. Neff, "A multimodal motion-captured corpus of matched and mismatched extravert-introvert conversational pairs", Proceedings of the Tenth International Conference on Language Resources and Evaluation (LREC'16), (2016), 3469-3476.

[24] Hu, T., A. Xu, Z. Liu, et al., "Touch Your Heart: A Tone-aware Chatbot for Customer Care on Social Media”, arXiv:1803.02952 [cs], 2018.

[25] Webster, J., and R. Watson, "Analyzing the Past to Prepare for the Future: Writing a Literature Review", MIS Quarterly 26, 2002.

[26] McCrae, R.R., and P.T. Costa Jr, "Personality trait structure as a human universal.", American psychologist 52(5), 1997, pp. 509.

[27] Haslam, N., Introduction to personality and intelligence, Sage, 2007.

[28] Matz, S., Y.W.F. Chan, and M. Kosinski, "Models of personality", In Emotions and Personality in Personalized Services. Springer, 2016, 35-54.

[29] Feist, J., and G.J. Feist, "Theories of Personality", Yogyakarta: Pustaka Pelajar, 2009.

[30] Boyd, R.L., and J.W. Pennebaker, "Language-based personality: a new approach to personality in a digital world", Current Opinion in Behavioral Sciences 18, 2017, pp. 63-68.

[31] Mairesse, F., and M.A. Walker, "Towards personalitybased user adaptation: psychologically informed stylistic language generation", User Modeling and UserAdapted Interaction 20(3), 2010, pp. 227-278.

[32] Allport, G.W., "Personality: A psychological interpretation.", 1937.

[33] Mehrabian, A., and S. Ferris, "Inference of attitudes form nonverbal communication in two channel", Journal of Consulting Psychology 13, 1967, pp. 37-58.

[34] Mehrabian, A., and M. Wiener, "Decoding of inconsistent communications.", Journal of personality and social psychology 6(1), 1967, pp. 109.

[35] Nass, C., and K.M. Lee, "Does computer-synthesized speech manifest personality? Experimental tests of recognition, similarity-attraction, and consistencyattraction.", Journal of experimental psychology: applied 7(3), 2001, pp. 171.

[36] Weizenbaum, J., "ELIZA - a computer program for the study of natural language communication between man and machine", Communications of the ACM 9(1), 1966, pp. 36-45.

[37] Qiu, L., and I. Benbasat, "Evaluating anthropomorphic product recommendation agents: A social relationship perspective to designing information systems", Journal 
of management information systems 25(4), 2009, pp. $145-182$.

[38] Seymour, M., L.I. Yuan, A. Dennis, K. Riemer, and others, "Have We Crossed the Uncanny Valley? Understanding Affinity, Trustworthiness, and Preference for Realistic Digital Humans in Immersive Environments", Journal of the Association for Information Systems 22(3), 2021, pp. 9.

[39] Von Der Pütten, A.M., N.C. Krämer, and J. Gratch, "1 Whol's there? Can a Virtual Agent Really Elicit Social Presence?", 2009.

[40] Schroeder, J., and M. Schroeder, "Trusting in Machines: How Mode of Interaction Affects Willingness to Share Personal Information with Machines", Proceedings of the 51st Hawaii International Conference on System Sciences, (2018).

[41] Schmitt, A., N. Zierau, A. Janson, and J.M. Leimeister, "Voice as a Contemporary Frontier of Interaction Design", European Conference on Information Systems (ECIS), (2021).

[42] Epley, N., A. Waytz, and J.T. Cacioppo, "On seeing human: a three-factor theory of anthropomorphism.", Psychological review 114(4), 2007, pp. 864.

[43] Nass, C., and Y. Moon, "Machines and Mindlessness: Social Responses to Computers", Journal of Social Issues 56(1), 2000, pp. 81-103.

[44] Gnewuch, U., S. Morana, M.T. Adam, and A. Maedche, " The Chatbot is typing...'-The Role of Typing Indicators in Human-Chatbot Interaction", Proceedings of the 17th Annual Pre-ICIS Workshop on HCI Research in MIS, (2018), 0-5.

[45] Gnewuch, U., S. Morana, M. Adam, and A. Maedche, "Faster is not always better: understanding the effect of dynamic response delays in human-chatbot interaction", 2018.

[46] Aylett, M.P., A. Vinciarelli, and M. Wester, "Speech synthesis for the generation of artificial personality", IEEE transactions on affective computing 11(2), 2020, pp. 361-372.

[47] Lee, S., G. Lee, S. Kim, and J. Lee, "Expressing Personalities of Conversational Agents through Visual and Verbal Feedback", Electronics 8(7), 2019, pp. 794.

[48] Hanna, N., and D. Richards, "Measuring the effect of personality on human-iva shared understanding", Proceedings of the 2014 international conference on autonomous agents and multi-agent systems, (2014), $1643-1644$.

[49] Li, J., M.X. Zhou, H. Yang, and G. Mark, "Confiding in and listening to virtual agents: The effect of personality", Proceedings of the 22nd International Conference on Intelligent User Interfaces, (2017), 275 286.

[50] Wester, M., M. Aylett, M. Tomalin, and R. Dall, "Artificial personality and disfluency", Sixteenth Annual Conference of the International Speech Communication Association, (2015).

[51] Isbister, K., and C. Nass, "Consistency of personality in interactive characters: verbal cues, non-verbal cues, and user characteristics", International Journal of HumanComputer Studies 53(2), 2000, pp. 251-267.
[52] Ma, X., E. Yang, and P. Fung, "Exploring perceived emotional intelligence of personality-driven virtual agents in handling user challenges", The World Wide Web Conference, (2019), 1222-1233.

[53] Chen, Z., Y. Lu, M.P. Nieminen, and A. Lucero, "Creating a Chatbot for and with Migrants: Chatbot Personality Drives Co-Design Activities", Proceedings of the 2020 ACM Designing Interactive Systems Conference, (2020), 219-230.

[54] Akbar, F., T. Grover, G. Mark, and M.X. Zhou, "The Effects of Virtual Agents' Characteristics on User Impressions and Language Use", Proceedings of the 23rd International Conference on Intelligent User Interfaces Companion, (2018), 1-2.

[55] Chen, Y., A. Naveed, and R. Porzel, "Behavior and preference in minimal personality: A study on embodied conversational agents", International conference on multimodal interfaces and the workshop on machine learning for multimodal interaction, (2010), 1-4.

[56] Partala, T., V. Surakka, and J. Lahti, "Affective effects of agent proximity in conversational systems", Proceedings of the third Nordic conference on Humancomputer interaction, (2004), 353-356.

[57] Gnewuch, U., M. Yu, and A. Maedche, "The effect of perceived similarity in dominance on customer selfdisclosure to chatbots in conversational commerce", Proceedings of the 28th European Conference on Information Systems (ECIS 2020), (2020).

[58] Koda, T., and T. Ishioh, "Analysis of the Effect of Agent's Embodiment and Gaze Amount on Personality Perception", Proceedings of the 4th International Workshop on Multimodal Analyses Enabling Artificial Agents in Human-Machine Interaction, (2018), 1-5.

[59] Rayon, A., T. Gonzalez, and D. Novick, "Analysis of gesture frequency and amplitude as a function of personality in virtual agents", Proceedings of the Workshop on Multimodal Analyses enabling Artificial Agents in Human-Machine Interaction, (2016), 3-9.

[60] Hu, C., M.A. Walker, M. Neff, and J.E.F. Tree, "Storytelling agents with personality and adaptivity", International Conference on Intelligent Virtual Agents, Springer (2015), 181-193.

[61] Cafaro, A., H.H. Vilhjálmsson, T.W. Bickmore, D. Heylen, and D. Schulman, "First impressions in useragent encounters: the impact of an agent's nonverbal behavior on users' relational decisions", Proceedings of the 2013 international conference on Autonomous agents and multi-agent systems, (2013), 1201-1202.

[62] Mairesse, F., M.A. Walker, M.R. Mehl, and R.K. Moore, "Using Linguistic Cues for the Automatic Recognition of Personality in Conversation and Text", Journal of Artificial Intelligence Research 30, 2007, pp. 457-500.

[63] Robert, L.P., R. Alahmad, C. Esterwood, S. Kim, S. You, and Q. Zhang, "A Review of Personality in Human Robot Interactions", arXiv preprint arXiv:2001.11777, 2020.

[64] Seeger, A.-M., J. Pfeiffer, and A. Heinzl, "Texting with human-like conversational agents: Designing for anthropomorphism", Journal of the Association for Information Systems: JAIS, 2021, pp. tba. 


\section{Appendix}

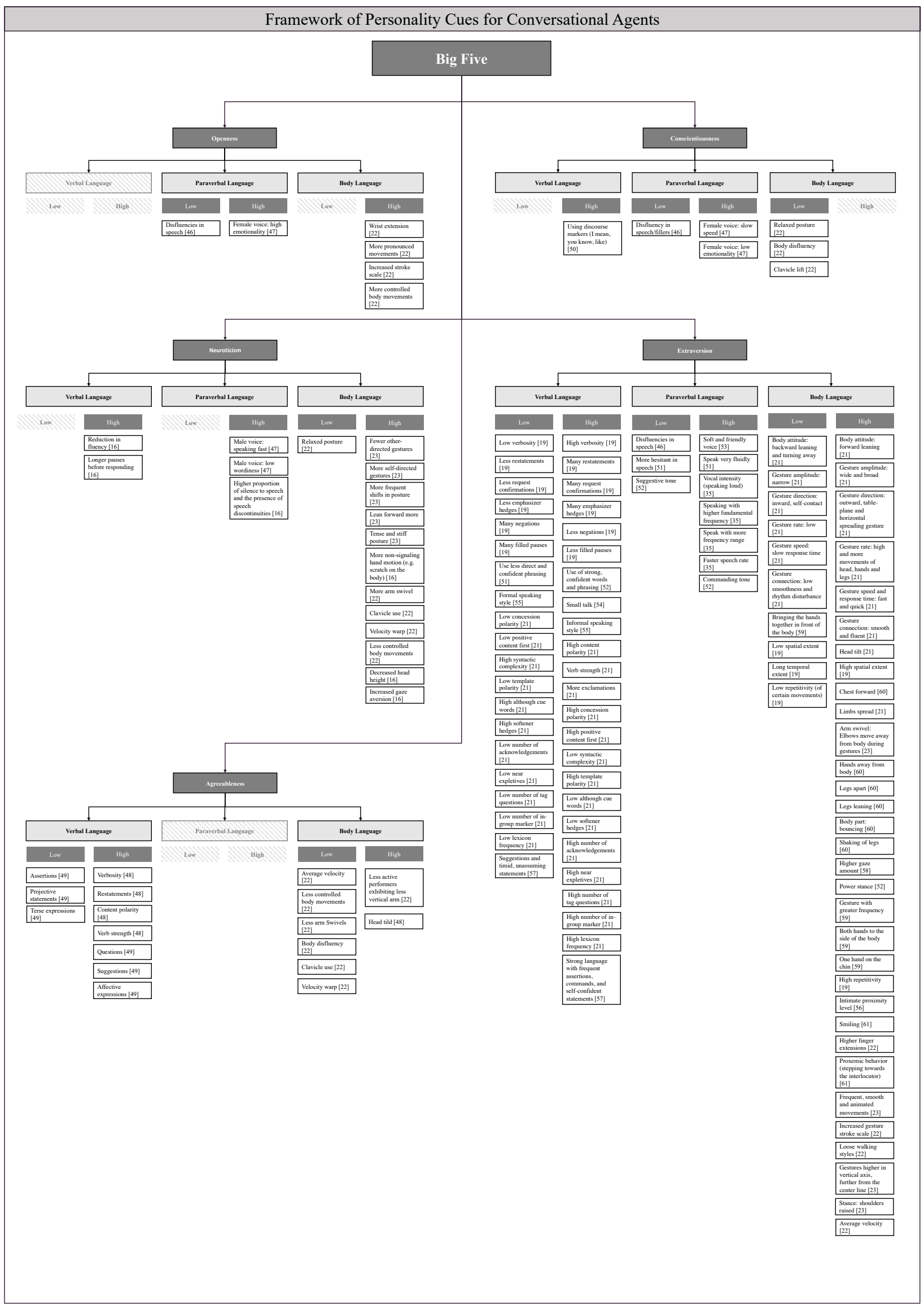

